\title{
Effects of suicide prevention videos developed by and targeting adolescents: a randomized controlled trial
}

\author{
Marlies Braun $^{1}$ (D) Benedikt Till ${ }^{1}$ D . Jane Pirkis ${ }^{2}$ (D) Thomas Niederkrotenthaler $^{1}$ (D)
}

Received: 21 June 2021 / Accepted: 8 November 2021

(c) The Author(s) 2021

\begin{abstract}
Suicide prevention videos featuring young people's personal narratives of hope and recovery are increasingly used in suicide prevention, but research on their effects is scarce. A double-blind randomized controlled trial was conducted to test the effects of a suicide prevention video featuring an adolescent mastering his suicidal ideation by getting help on 14 to 19-year-olds. $N=299$ adolescents were randomly allocated to watch the intervention video $(n=148)$ or a control video unrelated to mental health $(n=151)$. Questionnaire data were collected before $\left(T_{1}\right)$ and immediately after exposure $\left(T_{2}\right)$, and 4 weeks later $\left(T_{3}\right)$. Data were analyzed with a repeated-measures ANCOVA. The primary outcome was suicidal ideation, assessed with the Reasons for Living Inventory for Adolescents. Secondary outcomes were help-seeking intentions, attitudes towards suicide, stigmatization of suicidality, and mood. There was an immediate beneficial effect of the intervention on suicidal ideation ( $T_{2}$ mean change from baseline within intervention group $M_{\text {Change }}=-0.16$ [95\% CI -0.20 to -0.12 ], mean difference compared to control group $M_{\text {Diff }}=-0.09$ [95\% CI -0.15 to -0.03 ], $\eta_{\mathrm{p}}{ }^{2}=0.03$ ), which was not maintained at $T_{3}$. Participants reported significantly higher help-seeking intentions, which was maintained at 4-week follow-up. They also reported a sustained reduction of favorable attitudes to suicide. Effects on suicidal ideation were mediated by identification with the featured protagonist. Adolescents appear to benefit from suicide prevention narratives featuring personal stories from peers on coping with suicidal ideation and help-seeking.
\end{abstract}

Trial registration DRKS00017405; 24/09/19; retrospectively registered.

Keywords Suicide prevention $\cdot$ Media $\cdot$ Adolescents $\cdot$ Randomized controlled trial $\cdot$ Papageno effect

Thomas Niederkrotenthaler

thomas.niederkrotenthaler@meduniwien.ac.at

Marlies Braun

marlies.braun@meduniwien.ac.at

Benedikt Till

benedikt.till@meduniwien.ac.at

Jane Pirkis

j.pirkis@unimelb.edu.au

1 Unit Suicide Research \& Mental Health Promotion, Department of Social and Preventive Medicine, Center for Public Health, Medical University of Vienna, Kinderspitalgasse 15, 1090 Vienna, Austria

2 Centre for Mental Health, Melbourne School of Population and Global Health, The University of Melbourne, Melbourne, Australia

\section{Introduction}

Youth suicide is a major public health problem in many countries [1] and ranks consistently among the three most common causes of death among young people [2]. Consequently, considerable attention has been given to how youth suicide might best be combatted. One promising intervention path is media interventions [3]. In recent years, videos featuring personal stories of hope and recovery from suicidal crises have increasingly been used for prevention and education purposes. Several studies suggest that media stories featuring positive narratives of coping might reduce suicidal ideation $[4,5]$ and increase help-seeking intentions [6], the so-called Papageno effect [7]. However, little is known about the impact of these narratives for young people $[8,9]$. There are currently only three randomized controlled trials (RCTs) available that investigated media effects of this kind of messaging in young adults $[8,10,11]$, but results are inconsistent, and none of these studies have 
included young adolescents under the age of 18. This is in spite of the fact that this age group is confronted with youthspecific challenges, such as managing identity formation, peer pressure, and developmental changes [12, 13], and the need for tailored prevention strategies has been highlighted in the literature [12, 14]. Media stories explicitly targeting a young audience and providing opportunities for identification might resonate better with this group than other approaches [15]. Video campaigns might be a particularly powerful prevention approach for this age group, as young people commonly use video platforms, such as YouTube, as sources for information [16].

In the present study, we aimed to investigate short- and medium-term effects of a suicide prevention video developed by adolescents for other adolescents who experience suicidal ideation regarding several mental health-related outcomes, including suicidal ideation (primary outcomes), help-seeking intentions, attitudes to suicide, and suicide stigma (secondary outcomes). We hypothesized that the intervention video would have a beneficial impact on these outcomes. Further, in accordance with previous studies, we hypothesized that the effect on suicidal ideation would be stronger the more youth identified with the story [17, 18], and the more vulnerable they were to suicide $[4,11,18]$. We also explored gender differences.

\section{Methods}

\section{Participants}

We conducted a double-blind RCT. Participants were recruited from July $2019(01 / 07 / 19)$ to October 2020 $(12 / 10 / 20)$. Youth aged 14-19 years were invited to participate in a study on health-related awareness videos; more specific details on the aim of the study were not provided and there was no mention of suicide. Invitation flyers were provided at locations in Vienna, Austria, including schools, university events, and online. The announcement stated that participants were required to come to the study center twice, with a 4-week time span between visits, with a compensation of $10 €$ per visit. Due to the COVID-19 lockdown in spring 2020, participation via an online questionnaire was additionally established to allow those who were already enrolled to complete their 4-week follow-up online.

At the beginning of the trial, inclusion criteria (i.e., age 14-19 years, good German skills, and Austrian residency/ permanently living in Austria) were checked, and included individuals completed the Beck Hopelessness Scale [19, 20] to determine individual vulnerability to suicide. Hopelessness has been found to be a robust predictor of suicide risk $[21,22]$. For participants who scored above the cut-off score of 33 , the researcher further assessed their well-being and immediate suicide risk and informed them about available help services. No participant was excluded in this process. Individual written informed consent was sought from youth and, for minors (under 18-year-olds), additionally from parents.

\section{Randomization and blinding}

Participants were provided with access to a computer workstation. Participants completed the questionnaire on a computer in the online tool SoSci Survey (www.soscisurvey.de). Participants were randomly allocated to view the intervention or control video. They were randomized individually using an automated algorithm in the online tool SoSci Survey. The algorithm applied urn randomization, i.e., a simple 1:1 randomization based on a computerized algorithm (https://www.soscisurvey.de/help/doku.php/en:create:random_urns) [23].

\section{Materials and procedure}

\section{Intervention and control video}

We tested a suicide prevention video that was produced by students within a school project in 2018/2019, supervised by the lead researcher [24]. In this project, 18 high school students aged 15-19 years produced suicide prevention videos in teams. In total, seven short films were produced. All videos featured young people engaging in various sorts of help-seeking. An international jury of suicide prevention experts selected the best video based on predefined quality criteria, and this video was the intervention video. For further details see Braun et al. [24], https://econtent.hogre fe.com/doi/suppl/10.1027/0227-5910/a000696/suppl_file/ 0227-5910_a000696_esm2.mp4.

The video had a duration of $4.51 \mathrm{~min}$ and featured a 17 -year-old boy telling his personal story about his past suicidal crisis and his way of mastering it. The protagonist describes the circumstances that contributed to his suicidal crisis, putting emphasis on how he came through it, noting the relevance of proactive help-seeking, and highlighting different sources of help (i.e., teacher, friends, and professional help). At the end of the video, a list of mental health services for youth was presented.

The control video had a duration of $3.54 \mathrm{~min}$ and focused on a personal narrative about a topic unrelated to mental health. It featured the same protagonist talking about nutrition, how he stays fit, and maintains a healthy lifestyle. At the end of the video, a list of references about nutrition and healthy lifestyle was presented. The intervention and the control video were similar in terms of style and production (see Braun et al. [24] for details). 


\section{Procedure}

Before watching the video $\left(T_{1}\right)$, socio-demographics, suicidal ideation, and all secondary outcomes (i.e., help-seeking intentions, attitudes towards suicide, stigma of suicide, and mood) were measured. Immediately after video exposure $\left(T_{2}\right)$, all outcomes were measured again and individuals' identification with the protagonist was assessed. All participants received a link to their video via e-mail, were encouraged to rewatch the video, and got an e-mail reminder every week. The number of individual viewings was automatically recorded in a database. At 4-week follow-up $\left(T_{3}\right)$, all outcome measures were assessed again, including an item to assess blinding success.

\section{Primary outcome measure}

\section{Suicidal ideation}

Suicidal ideation was assessed with the Reasons for Living Inventory for Adolescents (RFL-A [25]). It consists of 32 self-report items (e.g., "I am afraid of killing myself") evaluating a range of adaptive beliefs and reasons for living. A rating scale from 1 (not at all important) to 6 (extremely important) was used. The RFL-A has been identified as a valid and reliable measure of adolescent suicide risk potential [26], and several versions of the RFL have been used in similar media-related studies $[4,10,18]$. Scores were reverse-coded, with higher scores indicating higher suicidal ideation (Cronbach's $\alpha=0.93$ ).

\section{Secondary outcome measures}

\section{Help-seeking intentions}

The 10-item General Help-seeking Questionnaire (GHSQ [27]) asks respondents to indicate the likelihood of seeking help in the case of suicidal thoughts from a variety of sources. Items were rated on a Likert scale from 1 (extremely unlikely) to 7 (extremely likely) (overall sum core: Cronbach's $\alpha=0.67$ ) and included subscales for private (4 items, Cronbach's $\alpha=0.71)$ and professional help-seeking intentions ( 3 items, Cronbach's $\alpha=0.70$ ). Higher scores indicate greater help-seeking intentions.

\section{Attitudes towards suicide}

Attitudes towards suicide were measured with the Cognitions Concerning Suicide Scale (CCSS [28, 29]). Respondents rated their level of agreement with 20 statements (e.g., "Everyone has the right to commit suicide") on a Likert scale from 1 (disagree) to 6 (agree). Higher scores indicate more favorable attitudes toward suicide (Cronbach's $\alpha=0.83$ ).

\section{Stigma of suicide}

The 12-item short version of the Stigma of Suicide Scale (SOSS [30]) was used to assess stigma. Items were rated on a Likert scale from 1 (strongly disagree) to 5 (strongly agree). Scores for the two subscales "Stigma" (8 items, Cronbach's $\alpha=0.87$ ) and "Glorification/Normalization" (4 items, Cronbach's $\alpha=0.73$ ) were calculated. Higher scores indicate greater stigmatizing attitudes toward suicidal individuals.

\section{Mood}

Current mood was assessed using the"Mood" subscale of the Affective State Scale (ASS [31]), which uses responses to eight adjectives describing respondents' mood, such as "merry" or "sad", scored on a scale from 1 (not at all) to 4 (highly). Higher scores indicate better mood (Cronbach's $\alpha=0.87$ ).

\section{Additional measures}

\section{Vulnerability}

The Beck Hopelessness Scale by Beck and Steer [19], a 10-item measure to assess hopelessness (e.g., "My future seems dark to me"), was used to assess vulnerability at $T_{1}$. Based on Krampen [20], a scale rating items from 1 (very false) to 6 (very true) was used. In accordance with previous studies [11], this variable was used to assess vulnerability to suicide. For this purpose, the sample was stratified into low vs. higher vulnerability based on the sample mean (Cronbach's $\alpha=0.77$ ).

\section{Identification}

Identification was assessed with Cohen's Identification Scale [32]. Respondents rated their level of agreement with 10 statements (e.g., "While watching the video I could feel the emotions of character X") on a scale from 1 (completely disagree) to 5 (completely agree). Higher scores indicate greater identification with the protagonist featured in the video (Cronbach's $\alpha=79$ ).

\section{Blinding success}

To assess blinding success, respondents were asked at $T_{3}$ to indicate what group they thought they had been allocated 
to ("intervention group", "control group", or "don't know") $[4,33]$.

\section{Power analysis}

The required sample size was calculated for a repeatedmeasures analysis of covariance (ANCOVA) with three measurements, a power of 0.80 , a two-sided significance level of $p<0.05$. We had $8(2 \times 2 \times 2)$ statistical groups (i.e., two study groups: intervention versus control; two groups for identification with the protagonist: low [i.e., below the sample mean] versus high [i.e., above the sample mean]; and two groups for suicide baseline vulnerability: low [i.e., below the sample mean] versus high [i.e., above the sample mean]). Assuming a correlation of 0.79 between measuring points, 288 participants were required to detect a moderate intervention effect on suicidal ideation (Cohen's $f=0.21$ ) $[5,11,18]$.

\section{Data analysis}

Mean scores were calculated for each outcome variable. Differences in the primary outcome (i.e., suicidal ideation) and in the secondary outcomes between intervention and control group were calculated using a repeated-measures analysis of covariance (ANCOVA). The models were controlled for age, gender, and for baseline scores. Bonferroni-adjusted contrast tests were used to compare individual group differences.

Differences in males and females were explored by adding gender as between-subjects independent variable to the model. To examine the effects of individuals' vulnerability and identification, the dichotomized variables for suicide baseline vulnerability and identification were added as between-subjects independent variables to the model. Further, a mediation analysis using PROCESS [34] was conducted to assess whether identification with the protagonist mediated the effect of the intervention. Model 4 and a bootstrapping method with 5000 bootstrap resamples was used. The bootstrapping method produced $95 \%$ bias-corrected confidence intervals of these effects [34].

\section{Sensitivity analysis}

To check whether the findings were different in minors (i.e., participants under the age of 18), a sensitivity analysis was conducted by reanalyzing the data in this subset of participants. Patterns in 14- to 17-year-olds were similar to the presented findings in the full sample (results available on request).

\section{Ethics statement}

All procedures contributing to this work comply with the ethical standards of the relevant national and institutional committees on human experimentation and with the Helsinki Declaration of 1975, as revised in 2008. All procedures involving human participants were approved by the research ethics board at the Medical University of Vienna (study protocol 1033/2015). Individual written consent was sought from all participants and for minors additionally from parents. Trial registration: German Clinical Trial Registry (DRKS00017405; 24 September 2019; retrospectively registered).

\section{Results}

\section{Study participant characteristics}

In total, 299 individuals were randomized (intervention group: $n=148$ and control group: $n=151)$. $N=9$ individuals did not provide data at 4-week follow-up, resulting in 290 individuals who completed the questionnaire $(96.99 \%)$ and were analyzed (see study flowchart: Fig. 1). Out of the 299 participants, $n=225$ participants were female $(75.25 \%)$ and $n=202$ participants $(67.56 \%)$ had Austrian nationality. The mean age was 17.95 years $(\mathrm{SD}=1.19)$, ranging from $14 \mathrm{up}$ to 19 years, with $n=82(27.42 \%)$ individuals being under 18 years old (see Table 1$) . N=53(17.73 \%)$ participants scored above the cut-off score at the baseline $\left(T_{1}\right)$ screening for vulnerability to suicide. $N=39(13.45 \%)$ individuals completed their participation at $T_{3}$ online. $N=46(15.38 \%)$ participants watched the video more than once. Baseline characteristics of participants were similar between groups as indicated by $\chi^{2}$ and independent $t$ tests (Table 1). Participants in the intervention group reported more favorable attitudes toward suicide at baseline, identified more with the portrayed character, and were more successful in guessing their group allocation correctly.

\section{Differences between dropouts and study completers}

We used independent $t$ tests and Fisher's exact tests to analyze whether participants who dropped out $(n=9)$ were different from study completers $(n=200)$. Austrian nationality $\left(\chi^{2}(2)=6.16, p=0.04\right)$, completed high school degree $\left(\chi^{2}(2)=9.51, p<0.01\right)$, and high school students $\left(\chi^{2}(2)=10.49, p<0.01\right)$ were underrepresented among dropouts with no further differences in socio-demographics or baseline $\left(T_{1}\right)$ measurements of outcome variables. 


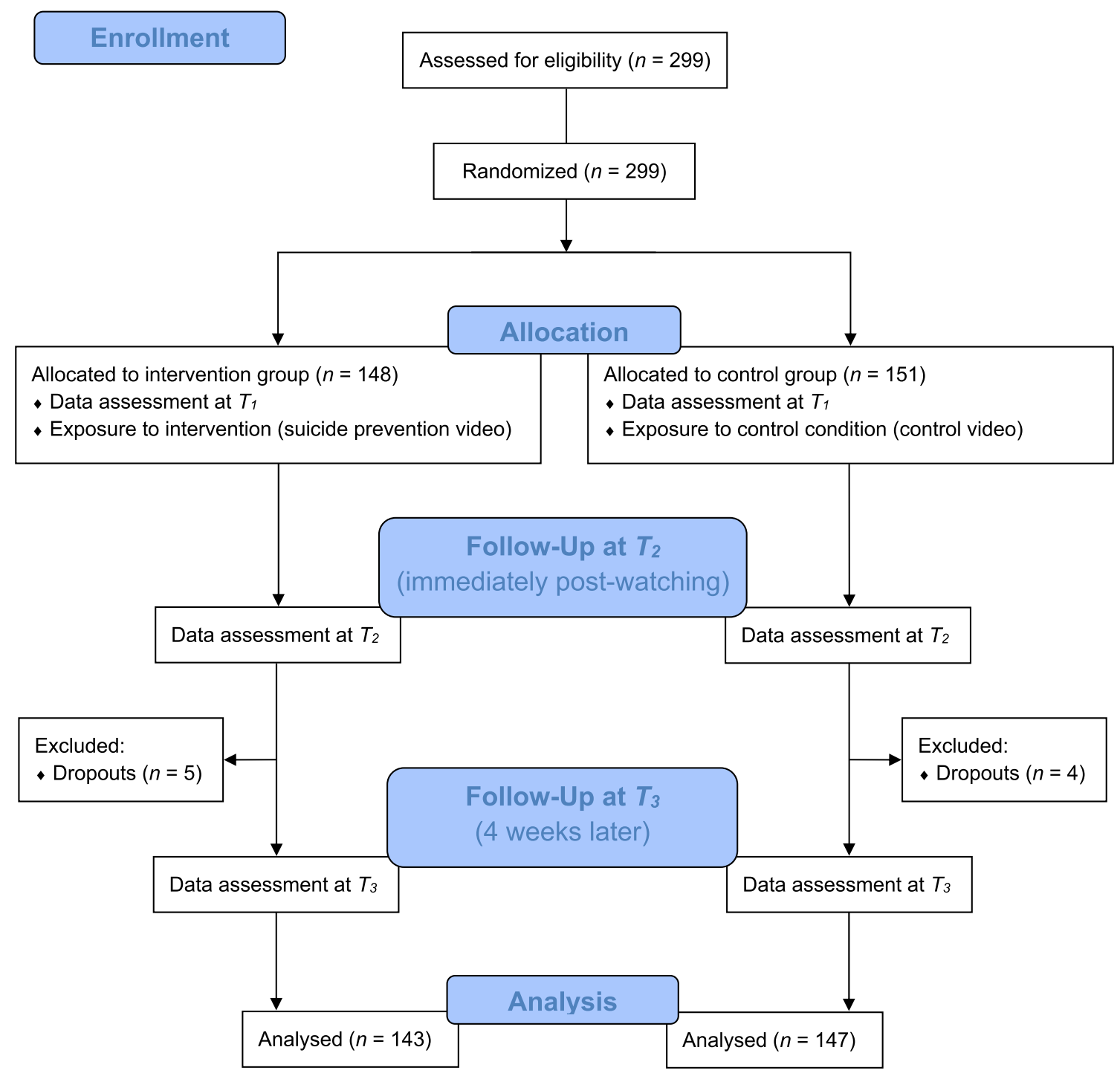

Fig. 1 Study flowchart

\section{Blinding}

86 participants (29.66\%) guessed their group allocation correctly, 87 (30.00\%) were incorrect, and 117 (40.34\%), responded with "don't know". More than half of the participants in each group were either uncertain or incorrect about their group assignment, with the majority of participants responding with "don't know" in both, intervention and control group respectively. There were no differences between the two study arms in correctly guessing the group allocation $\left(\chi^{2}(1)=3.81, p>0.05\right)$.

\section{Suicidal ideation}

Table 2 compares outcomes in both study arms. Suicidal ideation scores at $T_{2}$ were significantly lower in the intervention group ( $T_{2}$ mean change from baseline within intervention group $M_{\text {Change }}=-0.16$ [ $95 \% \mathrm{CI}-0.20$ to -0.12 ], mean difference compared with control group $\mathrm{M}_{\text {Diff }}=-0.09[95 \%$ CI -0.15 to -0.03$\left.], \eta_{p}{ }^{2}=0.03\right)$. This small effect was not maintained at 4-week follow-up $\left(T_{3} M_{\text {Change }}=-0.09[95 \%\right.$ $\mathrm{CI}-0.16$ to -0.03$], \mathrm{M}_{\text {Diff }}=-0.09$ [95\% CI -0.19 to 0.00$]$ ). Findings for the suicidal ideation subscale Peer Acceptance and Support revealed a similar pattern, with significantly lower scores in the intervention group $\left(T_{2} M_{\text {Change }}=-0.25\right.$ [95\% CI -0.33 to -0.17 ], $M_{\text {Diff }}=-0.20$ [95\% CI -0.30 to -0.10$], \eta_{\mathrm{p}}{ }^{2}=0.05$ ), which was maintained 4 weeks later $\left(T_{3} M_{\text {Change }}=-0.08\right.$ [ $95 \% \mathrm{CI}-0.18$ to 0.01$], M_{\text {Diff }}=-0.18$ [95\% CI -0.32 to -0.04$\left.], \eta_{\mathrm{p}}^{2}=0.02\right)$. 
Table 1 Descriptive statistics for intervention group $(n=148)$ and control group $(n=151)$

\begin{tabular}{|c|c|c|c|}
\hline Variable & $\begin{array}{l}\text { Group \#1: interven- } \\
\text { tion group }\end{array}$ & Group \#2: control group & $\chi^{2} / T$ \\
\hline \multicolumn{4}{|l|}{ Gender } \\
\hline Females $n(\%)$ & $114(77.03)$ & $111(73.51)$ & \multirow[t]{2}{*}{$0.50^{\mathrm{a}}$} \\
\hline Males $n(\%)$ & $34(22.97)$ & $40(26.49)$ & \\
\hline Age $M(S D)$ & $17.99(1.21)$ & $17.91(1.18)$ & $0.57^{\mathrm{c}}$ \\
\hline \multicolumn{4}{|l|}{ Nationality } \\
\hline Austrians $n(\%)$ & $105(70.95)$ & $97(64.24)$ & \multirow[t]{3}{*}{$1.82^{\mathrm{b}}$} \\
\hline Germans $n(\%)$ & $9(6.08)$ & $9(5.96)$ & \\
\hline Other nationality $n(\%)$ & $34(22.97)$ & $45(29.80)$ & \\
\hline \multicolumn{4}{|l|}{ Occupation } \\
\hline Student (school) $n(\%)$ & $63(42.57)$ & $69(45.70)$ & \multirow[t]{3}{*}{$2.80^{\mathrm{b}}$} \\
\hline Student (university) $n(\%)$ & $69(46.62)$ & $58(38.41)$ & \\
\hline Other occupation $n(\%)$ & $16(10.81)$ & $24(15.89)$ & \\
\hline Suicide attempt in last year $n(\%)$ & $12(8.11)$ & $8(5.30)$ & $0.95^{\mathrm{a}}$ \\
\hline Treatment of mental illness at present $n(\%)$ & $4(2.70)$ & $7(4.64)$ & $0.79^{\mathrm{a}}$ \\
\hline \multicolumn{4}{|l|}{ Recruitment method } \\
\hline Recruitment via website $n(\%)$ & $60(40.54)$ & $72(47.68)$ & \multirow[t]{3}{*}{$1.77^{\mathrm{b}}$} \\
\hline Recruitment via recommendation $n(\%)$ & $26(17.57)$ & $26(17.22)$ & \\
\hline Recruitment via other sources $n(\%)$ & $62(41.89)$ & $53(35.10)$ & \\
\hline Baseline hopelessness (BHS) $M$ (SD) & $2.57(0.69)$ & $2.59(0.75)$ & $-0.21^{\mathrm{c}}$ \\
\hline Baseline suicidal ideation (RFL-A total) $M(\mathrm{SD})$ & $2.34(0.79)$ & $2.35(0.75)$ & $-0.11^{\mathrm{c}}$ \\
\hline Baseline Family Alliance (RFL-A subscale) $M$ (SD) & $2.18(1.22)$ & $2.19(1.15)$ & $-0.05^{\mathrm{c}}$ \\
\hline Baseline Suicide Related Concerns (RFL-A subscale) $M(\mathrm{SD})$ & $2.89(1.38)$ & $2.97(1.45)$ & $-0.48^{\mathrm{c}}$ \\
\hline Baseline Peer Acceptance and Support (RFL-A subscale) $M$ (SD) & $2.18(0.98)$ & $2.07(0.97)$ & $0.93^{\mathrm{c}}$ \\
\hline Baseline help-seeking (GHSQ total) $M$ (SD) & $3.70(1.08)$ & $3.83(1.04)$ & $-1.09^{\mathrm{c}}$ \\
\hline Baseline help-seeking private (GHSQ subscale) $M(\mathrm{SD})$ & $4.03(1.33)$ & $4.18(1.25)$ & $-1.00^{\mathrm{c}}$ \\
\hline Baseline help-seeking professional (GHSQ subscale) $M$ (SD) & $3.62(1.35)$ & $3.63(1.36)$ & $-0.08^{\mathrm{c}}$ \\
\hline Baseline attitudes toward suicide (CCSS) $M(\mathrm{SD})$ & $2.63(0.76)$ & $2.45(0.75)$ & $2.13^{\mathrm{cd}}$ \\
\hline Baseline stigma towards suicide (SOSS subscale) $M(\mathrm{SD})$ & $2.17(0.89)$ & $2.16(0.88)$ & $0.08^{\mathrm{c}}$ \\
\hline Baseline normalization/glorification of suicide (SOSS subscale) $M(\mathrm{SD})$ & $2.04(0.86)$ & $1.99(0.81)$ & $0.50^{\mathrm{c}}$ \\
\hline Baseline mood (ASS) $M(\mathrm{SD})$ & $2.99(0.59)$ & $3.03(0.60)$ & $-0.48^{\mathrm{c}}$ \\
\hline Identification with protagonist featured in video $M(S D)$ & $3.56(0.74)$ & $3.38(0.67)$ & $2.30^{\mathrm{cd}}$ \\
\hline Multiple video viewing $n(\%)$ & $24(16.22)$ & $22(14.57)$ & $0.16^{\mathrm{a}}$ \\
\hline Completed questionnaire $n(\%)$ & $143(96.62)$ & $147(97.35)$ & $0.14^{\mathrm{a}}$ \\
\hline Manipulation check correct $n(\%)$ & $50(34.97)$ & $36(24.49)$ & $7.37^{\text {bd }}$ \\
\hline
\end{tabular}

Frequencies $(n)$, percentages (\%), means $(M)$, and standard deviations (SD) provided for each group, as well as $\chi^{2}$ values from $\chi^{2}$ tests and $t$ values from independent $t$ tests testing group differences

${ }^{\mathrm{a}} \chi^{2}$ test result. $d f=1$

${ }^{\mathrm{b}} \chi^{2}$ test result. $d f=2$

${ }^{\mathrm{c}}$ Independent $t$ test result

${ }^{\mathrm{d}} p<0.05$

\section{Secondary outcomes}

Participants in the intervention group reported significantly higher likelihood of seeking help in general at $T_{2}\left(T_{2}\right.$ $M_{\text {Change }}=0.22$ [95\% CI 0.13-0.31], $M_{\text {Diff }}=0.20$ [95\% CI $\left.0.10-0.30], \eta_{\mathrm{p}}{ }^{2}=0.05\right)$. This small effect was not maintained at 4-week follow-up $\left(T_{3} M_{\text {Change }}=0.30\right.$ [95\% CI 0.15-0.45],
$M_{\text {Diff }}=0.15$ [95\% CI -0.04 to 0.33 ). There was an immediate significant small effect on help-seeking intentions from private sources $\left(T_{2} M_{\text {Change }}=0.27\right.$ [95\% CI 0.15-0.39], $M_{\text {Diff }}=0.20$ [95\% CI 0.06-0.34], $\eta_{\mathrm{p}}{ }^{2}=0.03$ ), which not maintained at 4-week follow-up $\left(T_{3} M_{\text {Change }}=0.34[95 \% \mathrm{CI}\right.$ $0.16-0.52$ ], $M_{\text {Diff }}=0.11$ [95\% CI -0.11 to 0.33 ). Participants in the intervention group also had higher help-seeking 


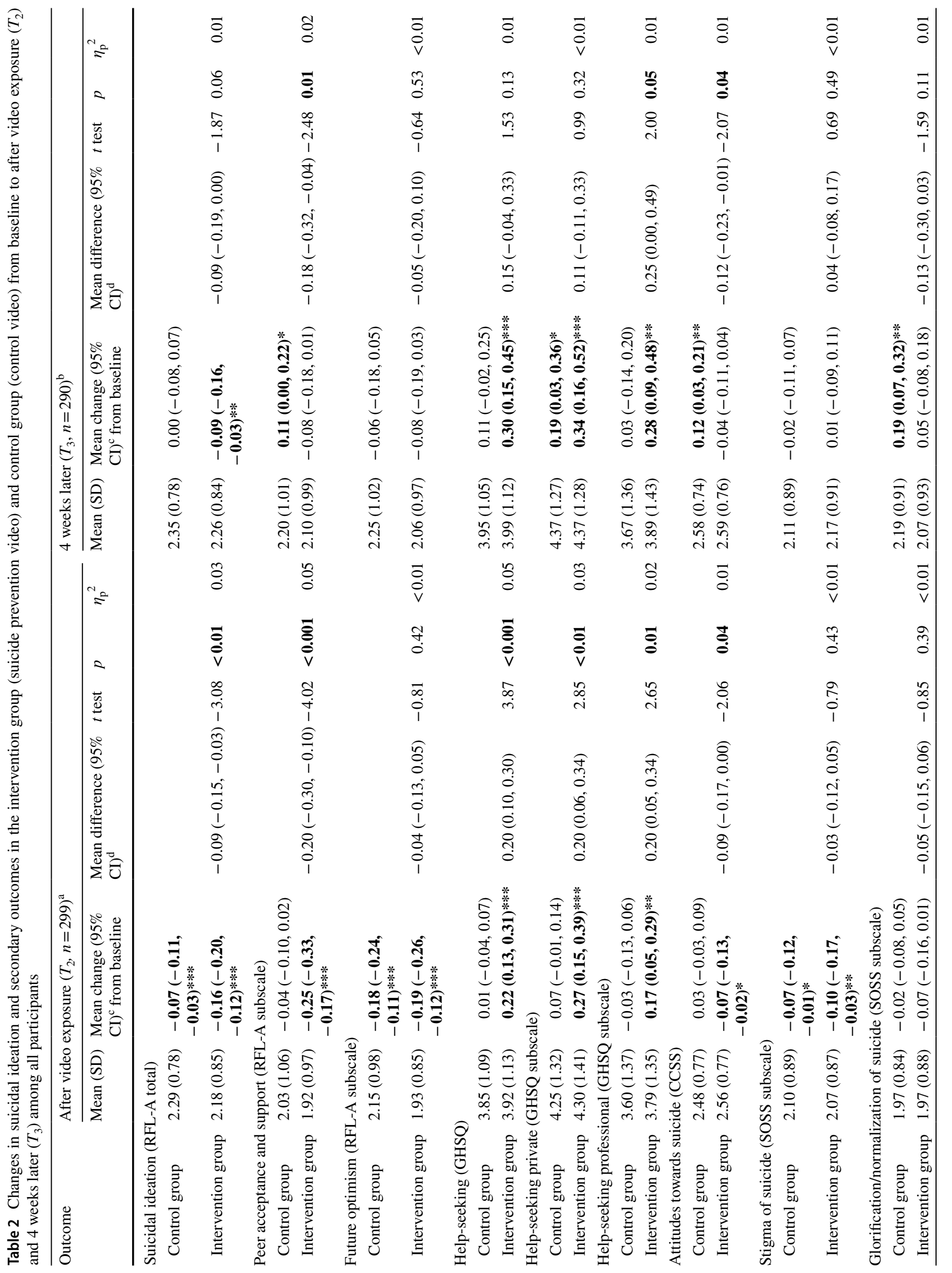




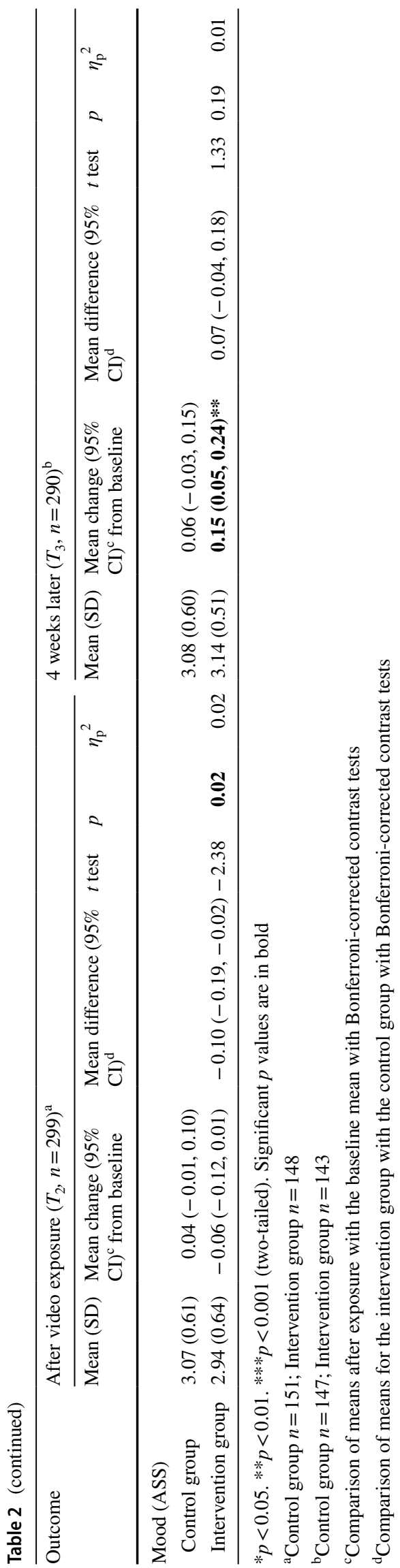

intentions from professional sources $\left(T_{2} M_{\text {Change }}=0.17\right.$ [95\% CI 0.05-0.29], $M_{\text {Diff }}=0.20$ [95\% CI $0.05-0.34$ ], $\left.\eta_{\mathrm{p}}{ }^{2}=0.02\right)$, which was maintained at 4-week follow-up $\left(T_{3}\right.$ $M_{\text {Change }}=0.28$ [95\% CI 0.09-0.48], $M_{\text {Diff }}=0.25$ [95\% CI $0.00-0.49], \eta_{\mathrm{p}}{ }^{2}=0.01$ ).

Participants in the intervention group showed a small decrease in favorable attitudes towards suicide $\left(T_{2}\right.$ $M_{\text {Change }}=-0.07$ [95\% CI -0.13 to -0.02 ], $M_{\text {Diff }}=-0.09$ [95\% CI -0.17 to 0.00$\left.], \eta_{\mathrm{p}}{ }^{2}=0.01\right)$. This effect was maintained at 4-week follow-up $\left(T_{3} M_{\text {Change }}=-0.04[95 \% \mathrm{CI}\right.$ -0.11 to $0.04 M_{\text {Diff }}=-0.12$ [95\% CI -0.23 to -0.01 , $\left.\eta_{\mathrm{p}}{ }^{2}=0.01\right)$. There was a short-term deterioration in mood in the intervention group $\left(T_{2} M_{\text {Change }}=-0.06[95 \% \mathrm{CI}\right.$ -0.12 to 0.01 ], $M_{\text {Diff }}=-0.10$ [ $95 \% \mathrm{CI}-0.19$ to -0.02 ], $\eta_{\mathrm{p}}{ }^{2}=0.02$ ), which was not maintained at 4-week follow-up. There was no significant group effect on the stigmatization of suicide as well as glorification/normalization of suicide.

\section{Effects of vulnerability}

The analysis revealed no significant group $\times$ vulnerability interaction with regard to suicidal ideation.

\section{Effects of identification}

There was no significant group $\times$ identification interaction with regard to suicidal ideation. The mediation analysis revealed that the relationship between group allocation and suicidal ideation at $T_{2}$ was mediated by identification (indirect effect IE $=0.03$ [95\% CI 0.001-0.08]). Allocation to the intervention group was associated with higher identification $(b=-0.19$, s.e. $=0.08, t(297)=-2.30, p=0.02)$, and identification was negatively associated with suicidal ideation $(b=-0.18$, s.e. $=0.07, t(297)=-2.68, p<0.01)$.

\section{Effects of gender}

We found no effect of gender with regard to suicidal ideation. The analysis revealed a significant group $\times$ gender interaction for intentions to help-seeking in general, $F(1,293)=5.21$, $p<0.05$ (see Table 3). Girls/women in the intervention group had higher help-seeking intentions $\left(T_{2} M_{\text {Change }}=0.26\right.$ [95\% CI 0.15-0.36], $M_{\text {Diff }}=0.27$ [95\% CI 0.15-0.39]). A similar pattern was present for help-seeking intentions from private sources $(F(1,293)=9.43, p<0.01)$, but not for helpseeking intentions from professional sources, which did not show any gender differences. 
Table 3 Changes in outcomes with a significant group $\times$ gender interaction in the intervention group (suicide prevention video) and control group (control video) from baseline to after video exposure $\left(T_{2}\right)$ among boys/men and girls/women

\begin{tabular}{|c|c|c|c|c|c|c|c|}
\hline Outcome, time after exposure & $\begin{array}{l}\text { Mean (SD) } \\
\text { after expo- } \\
\text { sure }\end{array}$ & $\begin{array}{l}\text { Mean change ( } 95 \% \\
\mathrm{CI})^{\mathrm{c}} \text { from baseline }\end{array}$ & Mean difference $(95 \% \mathrm{CI})^{\mathrm{d}}$ & $t$ test & $p$ & $\eta_{\mathrm{p}}^{2}$ & $\begin{array}{l}\text { Group } \times \text { Gender } \\
F \\
p^{e}\end{array}$ \\
\hline \multicolumn{8}{|l|}{ Help-seeking (GHSQ), $T_{2}$} \\
\hline Male $^{\mathrm{a}}$ & & & & & & & 5.21 \\
\hline Control group & $4.28(0.85)$ & $0.08(-0.01,0.18)$ & $0.00(-0.18,0.19)$ & 0.05 & 0.96 & $<0.01$ & 0.02 \\
\hline Intervention group & $3.76(1.25)$ & $0.10(-0.06,0.26)$ & & & & & \\
\hline \multicolumn{8}{|l|}{ Female $^{\mathrm{b}}$} \\
\hline Control group & $3.69(1.13)$ & $-0.01(-0.08,0.05)$ & $0.27(0.15,0.39)$ & 4.35 & $<0.001$ & 0.08 & \\
\hline Intervention group & $3.97(1.09)$ & $0.26(0.15,0.36)^{* * *}$ & & & & & \\
\hline \multicolumn{8}{|c|}{ Help-seeking private (GHSQ subscale), $T_{2}$} \\
\hline Male $^{\mathrm{a}}$ & & & & & & & 9.43 \\
\hline Control group & $5.05(0.90)$ & $0.28(0.14,0.42)^{* * *}$ & $-0.22(-0.48,0.04)$ & -1.69 & 0.09 & 0.04 & $<0.01$ \\
\hline Intervention group & $4.02(1.56)$ & $0.16(-0.05,0.38)$ & & & & & \\
\hline \multicolumn{8}{|l|}{ Female $^{\mathrm{b}}$} \\
\hline Control group & $3.96(1.33)$ & $-0.01(-0.09,0.08)$ & $0.32(0.16,0.48)$ & 3.94 & $<0.001$ & 0.07 & \\
\hline Intervention group & $4.38(1.35)$ & $0.30(0.16,0.44) * * *$ & & & & & \\
\hline
\end{tabular}

${ }^{*} p<0.05 . * * p<0.01 . * * * p<0.001$ (two-tailed). Significant $p$ values are in bold

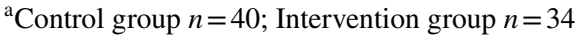

${ }^{\mathrm{b}}$ Control group $n=111$; Intervention group $n=114$

${ }^{\mathrm{c}}$ Comparison of means after exposure with the baseline mean with Bonferroni-corrected contrast tests

${ }^{\mathrm{d}}$ Comparison of means for the intervention group with the control group with Bonferroni-corrected contrast tests

${ }^{\mathrm{e}} \mathrm{ANCOVA}$ results, $d f=1$

\section{Discussion}

This study is the first to assess the effects of a short suicide prevention video developed by and targeting adolescents in a randomized controlled trial. Young people benefitted from the brief intervention that included watching a short film featuring an adolescent with past suicidal ideation, describing his story of getting help and recovering. Adolescents reported significantly lower suicidal ideation after watching the intervention video. The video was also effective in increasing help-seeking intentions, which has been highlighted as an essential target domain for suicide prevention [3]. We found that the video increased help-seeking intentions, including from private and professional sources, and the effects were maintained until 4-week follow-up. Immediate effects on help-seeking intentions were present in girls/ women but not in boys/men, which is consistent with previous studies, suggesting that males show lower help-seeking $[6,35]$. There were, however, no gender differences for the crucial aspect of help-seeking from professional sources, indicating that also boys/men benefitted to some extent in terms of help-seeking intentions. Furthermore, favorable attitudes towards suicide that have previously been associated with higher suicide risk [29], decreased significantly after watching the suicide prevention video.
The findings build on and extend findings from previous studies which suggest a reduction of suicidal ideation after exposure to media stories featuring individuals telling their stories of mastering their crises $[4,5,11,18]$. In this study, individuals with varying levels of vulnerability to suicide benefitted from the prevention videos. Compared to previous research in the area, we identified a more than seven times higher proportion of individuals with vulnerability to suicide $(N=53 ; 17.73 \%)$ using the same measure and cut-off score for vulnerability [11]. This is important because some previous studies in the field have found positive effects on suicidal ideation particularly in individuals with some degree of vulnerability to suicide $[4,10,11,18]$. Although this is encouraging regarding positive effects in young people who are at risk, a current limitation of the available research including the present study is that it focused on individuals from the general population and all studies, except the present one, included adults $[4,10,11,18]$. This means that the findings cannot be generalized to young individuals with clinical suicidality. Future studies should aim to assess effects of stories of coping and recovery in young people with some degree of vulnerability based on clinical assessment. Effects in these groups might deviate from the present findings, and specific circumstances and diagnoses might be relevant to any effects.

Unlike previous studies that focused on harmful media impacts of stories of suicide [18], the reduction in suicidal 
ideation following exposure to the suicide prevention video was mediated by identification. In general, identification with the narrative was high in the intervention group and higher compared to a previous study using the same measure for identification [15] - this might be because the videos were specifically tailored to adolescents and featured a young protagonist. The findings highlight the necessity of producing narratives that the audience can easily identify with to reduce suicidal ideation.

Although there are studies on personal media narratives of hope, which have found a media effect on either suicidal ideation [4, 5] or help-seeking [6], this is the first study that detected preventive effects on both of these crucial outcomes. This finding indicates that it is indeed possible to positively influence both outcomes with the same narrative. The present narrative emphasized both, the individual mastery of the suicidal crisis and the proactive help-seeking and success of help-seeking. At one point, the male protagonist encourages the audience to do the same by saying: "It is important to seek help [...]. To get rid of those [suicidal] thoughts, you have to talk to someone. [...] Now I know that every human life matters. Mine too. [...] It takes a lot of time and effort. However, it pays off". This clearly links his personal mastery of suicidal ideation to his help-seeking efforts.

\section{Strengths and limitations}

Strengths of this study include the RCT design with a sufficient number of participants and very low dropouts, and blinding was successful. Furthermore, the study was conducted on-site rather than online, increasing its validity [36], and differences between the intervention and the control video were minimal regarding stylistic means. Finally, the assessment included a 4-week follow-up to test for the sustainability of the effects, rather than only immediate effects like in previous studies $[5,10,18]$.

The study had some limitations. First, the sample size was only appropriate to detect medium-sized effects. Only a small number of participants watched the video multiple times, so we were not able to calculate effects of repeated exposure. Female adolescents were overrepresented in the study sample. Further, participants were exposed to one intervention video only, and materials should be tailored specifically to various groups, e.g., girls [37]. However, gender patterns suggested that girls/women did not feel less addressed by the male protagonist, but scored better on helpseeking intentions than boys/men. Outcome measures did not capture actual behavior (i.e., suicidal behavior or helpseeking behaviors), which would require larger sample sizes and longer follow-up. Finally, the present sample included a number of youth with some degree of vulnerability to suicide, but it remains unknown if the effects generalize to clinical samples at risk of suicide.

\section{Conclusion}

Adolescents appear to benefit from personal video narratives featuring adolescents who master their suicidal crises and emphasize help-seeking, consistent with the Papageno effect [7]. Suicide prevention videos developed by and for adolescents appear to be safe and effective in reducing some risk factors for suicide. These findings should serve as a bold encouragement for public health and school authorities to implement similar projects that put young people in charge of the development of media interventions for suicide prevention.

Author contributions TN formulated the research question and conceptualized the study. TN, MB, and BT contributed to the design of the study. MB carried out the study. MB performed the statistical analysis, supervised by TN. MB wrote the first draft of the manuscript. MB, BT, $\mathrm{JP}$, and TN contributed to manuscript revision, read, and approved the submitted version.

Funding Open access funding provided by Medical University of Vienna. This project is funded by the FWF (Austrian Science Fund, project number: KLI 627).

Data availability The data from this study are available from the corresponding author upon reasonable request.

\section{Declarations}

Conflicts of interest The authors declare that they have no conflict of interest.

Ethics approval All procedures contributing to this work comply with the ethical standards of the relevant national and institutional committees on human experimentation and with the Helsinki Declaration of 1975 , as revised in 2008. All procedures involving human participants were approved by the research ethics board at the Medical University of Vienna (study protocol 1033/2015).

Consent to participate Individual written consent was sought from all participants and for minors additionally from parents.

Consent for publication The participants provided informed consent for publication of anonymized data.

Open Access This article is licensed under a Creative Commons Attribution 4.0 International License, which permits use, sharing, adaptation, distribution and reproduction in any medium or format, as long as you give appropriate credit to the original author(s) and the source, provide a link to the Creative Commons licence, and indicate if changes were made. The images or other third party material in this article are included in the article's Creative Commons licence, unless indicated otherwise in a credit line to the material. If material is not included in the article's Creative Commons licence and your intended use is not permitted by statutory regulation or exceeds the permitted use, you will need to obtain permission directly from the copyright holder. To view a copy of this licence, visit http://creativecommons.org/licenses/by/4.0/. 


\section{References}

1. World Health Organization (WHO) (2014) Preventing suicide: A global imperative [Internet]. World Health Organization, Geneva. http://www.who.int/mental_health/suicide-prevention/ world_report_2014/en/. Accessed 20 May 2021

2. World Health Organization (WHO) (2019) Suicide [Internet]. World Health Organization, Geneva. https://www.who.int/newsroom/fact-sheets/detail/suicide. Accessed 20 May 2021

3. Niederkrotenthaler T, Reidenberg DJ, Till B, Gould MS (2014) Increasing help-seeking and referrals for individuals at risk for suicide by decreasing stigma. Am J Prev Med 47(3):S235-S243

4. Niederkrotenthaler T, Till B (2019) Effects of suicide awareness materials on individuals with recent suicidal ideation or attempt: online randomised controlled trial. Br J Psychiatry 17:1-8

5. Till B, Arendt F, Scherr S, Niederkrotenthaler T (2019) Effect of educative suicide prevention news articles featuring experts with vs without personal experience of suicidal ideation: a randomized controlled trial of the Papageno effect. J Clin Psychiatry 80(1): $17 \mathrm{~m} 11975$

6. King KE, Schlichthorst M, Spittal MJ, Phelps A, Pirkis J (2018) Can a documentary increase help-seeking intentions in men? A randomised controlled trial. J Epidemiol Community Health 72(1):92-98

7. Niederkrotenthaler T, Voracek M, Herberth A, Till B, Strauss M, Etzersdorfer E et al (2010) Role of media reports in completed and prevented suicide: Werther v. Papageno effects. Br J Psychiatry 197(3):234-243

8. Ftanou M, Ross A, Machlin A, Spittal MJ, King K, Nicholas A et al (2020) Public service announcements to change attitudes about youth suicide: a randomized controlled trial. Arch Suicide Res 1:1-16

9. Klimes-Dougan B, Lee C-YS (2010) Suicide prevention public service announcements: perceptions of young adults. Crisis 31(5):247-254

10. Niederkrotenthaler T, Till B (2020) Effects of awareness material featuring individuals with experience of depression and suicidal thoughts on an audience with depressive symptoms: randomized controlled trial. J Behav Ther Exp Psychiatry 66:101515

11. Till B, Tran US, Voracek M, Niederkrotenthaler T (2017) Beneficial and harmful effects of educative suicide prevention websites: randomised controlled trial exploring Papageno $v$. Werther effects. Br J Psychiatry 211(2):109-115

12. Wasserman C, Hoven CW, Wasserman D, Carli V, Sarchiapone M, Al-Halabí S et al (2012) Suicide prevention for youth—a mental health awareness program: lessons learned from the Saving and Empowering Young Lives in Europe (SEYLE) intervention study. BMC Public Health 12(1):776

13. Zarrett N, Eccles J (2006) The passage to adulthood: challenges of late adolescence. New Dir Youth Dev 2006(111):13-28

14. van der Feltz-Cornelis CM, Sarchiapone M, Postuvan V, Volker D, Roskar S, Grum AT et al (2011) Best practice elements of multilevel suicide prevention strategies: a review of systematic reviews. Crisis 32(6):319-333

15. Niederkrotenthaler T, Arendt F, Till B (2015) Predicting intentions to read suicide awareness stories: the role of depression and characteristics of the suicidal role model. Crisis 36(6):399-406

16. Anderson M, Jiang J (2018) Teens, social media \& technology 2018 [Internet]. Pew Research Center: Internet and Technology. https://www.pewresearch.org/internet/2018/05/31/teens-socialmedia-technology-2018/. Accessed 20 May 2021

17. Niederkrotenthaler T, Till B, Kapusta ND, Voracek M, Dervic K, Sonneck G (2009) Copycat effects after media reports on suicide: a population-based ecologic study. Soc Sci Med 69(7):1085-1090
18. Till B, Strauss M, Sonneck G, Niederkrotenthaler T (2015) Determining the effects of films with suicidal content: a laboratory experiment. Br J Psychiatry 207(1):72-78

19. Beck AT, Steer RA (1988) Manual for the beck hopelessness scale. Psychological Corporation, San Antonio

20. Krampen G (1994) Skalen zur Erfassung von Hoffnungslosigkeit (H-Skalen). Deutsche Bearbeitung und Weiterentwicklung der H-Skalen von Aaron T. Beck [Scales for assessment of hopelessness (H-scales). German adaption and enhancement of the H-scales by Aaron T. Beck]. Hogrefe, Göttingen

21. Beck AT, Brown G, Berchick R, Stewart BL, Steer RA (1990) Relationship between hopelessness and ultimate suicide: a replication with psychiatric outpatients. Am J Psychiatry 147(2):190-195

22. McMillan D, Gilbody S, Beresford E, Neilly L (2007) Can we predict suicide and non-fatal self-harm with the Beck Hopelessness Scale? A Meta-analysis Psychol Med 37(6):769-778

23. Wei LJ, Lachin JM (1988) Properties of the urn randomization in clinical trials. Control Clin Trials 9(4):345-364

24. Braun M, Till B, Pirkis J, Niederkrotenthaler T (2020) Suicide prevention videos developed by and for adolescents: a qualitative study. Crisis 42(2):114-120

25. Osman A, Downs WR, Kopper BA, Barrios FX, Baker MT, Osman JR et al (1998) The reasons for living inventory for adolescents (RFL-A): development and psychometric properties. J Clin Psychol 54(8):1063-1078

26. Gutierrez PM, Osman A, Kopper BA, Barrios FX (2000) Why young people do not kill themselves: the reasons for living inventory for adolescents. J Clin Child Psychol 29(2):177-187

27. Wilson C, Deane F, Ciarrochi J, Rickwood D (2005) Measuring help-seeking intentions: properties of the general help-seeking questionnaire. Can J Couns 39(1):15-28

28. Biblarz A, Brown M, Biblarz DN, Pilgrim M, Baldree BF (1991) Media influence on attitudes toward suicide. Suicide Life Threat Behav 21(4):374-384

29. Cwik JC, Till B, Bieda A, Blackwell SE, Walter C, Teismann T (2017) Measuring attitudes towards suicide: preliminary evaluation of an attitude towards suicide scale. Compr Psychiatry 72:56-65

30. Batterham PJ, Calear AL, Christensen H (2013) The stigma of suicide scale: psychometric properties and correlates of the stigma of suicide. Crisis 34(1):13-21

31. Becker P (1988) Skalen für Verlaufsstudien der emotionalen Befindlichkeit [Scales for longitudinal studies of affective state]. Z Für Exp Angew Psychol 35:345-369

32. Cohen J (2001) Defining identification: a theoretical look at the identification of audiences with media characters. Mass Commun Soc 4(3):245-264

33. Kolahi J, Bang H, Park J (2009) Towards a proposal for assessment of blinding success in clinical trials: up-to-date review. Commun Dent Oral Epidemiol 37(6):477-484

34. Hayes AF (2013) Introduction to mediation, moderation, and conditional process analysis: a regression-based approach. The Guilford Press, New York, p 507 (Methodology in the social sciences)

35. Galdas PM, Cheater F, Marshall P (2005) Men and health helpseeking behaviour: literature review. J Adv Nurs 49(6):616-623

36. Wright KB (2005) Researching internet-based populations: advantages and disadvantages of online survey research, online questionnaire authoring software packages, and web survey services. J Comput-Mediat Commun 10(3)

37. Ruch DA, Sheftall AH, Schlagbaum P, Rausch J, Campo JV, Bridge JA (2019) Trends in suicide among youth aged 10 to 19 years in the United States, 1975 to 2016. JAMA Netw Open 2(5):e193886 